INPLASY

PROTOCOL

To cite: Xiong et al. Clinical efficacy and safety of Jingui Shenqi Pills in the treatment of diabetic kidney disease: a systematic review and metaanalysis. Inplasy protocol 2021100085. doi: 10.37766/inplasy2021.10.0085

Received: 22 October 2021

Published: 22 October 2021

Corresponding author:

Pingjie Xiong

936917144@qq.com

Author Affiliation:

Guangdong Pharmaceutical University;The First Affiliated Hospital of Guangdong

Pharmaceutical University.

Support: None.

Review Stage at time of this submission: Preliminary searches.

Conflicts of interest:

None declared.

\section{Clinical efficacy and safety of Jingui Shenqi Pills in the treatment of diabetic kidney disease: a systematic review and meta-analysis}

Xiong, PJ1; Liu, XY2; Huang, XQ33; Fang, WF4; Piao, SH5; Guo, J6.

Review question / Objective: The purpose of this systematic review is to evaluate the efficiency and safety of Jingui Shenqi Pills in the treatment of diabetic kidney disease.

Condition being studied: Diabetic kidney disease (DKD) is one of the most common complications of diabetes mellitus. Jingui Shenqi pills (JSP) is a Chinese patent medicine, It is widely used in China to treat DKD. At present, there are some reports about the treatment of DKD with JSP, but the conclusions are not consistent, However, there is no evidence-based medical evidence to prove its safety and effectiveness, and most of the research samples are small, which can not provide enough test efficiency. The purpose of this systematic review is to evaluate the efficiency and safety of JSP in the treatment of DKD.

INPLASY registration number: This protocol was registered with the International Platform of Registered Systematic Review and Meta-Analysis Protocols (INPLASY) on 22 October 2021 and was last updated on 22 October 2021 (registration number INPLASY2021100085).

\section{INTRODUCTION}

Review question / Objective: The purpose of this systematic review is to evaluate the efficiency and safety of Jingui Shenqi Pills in the treatment of diabetic kidney disease.
Condition being studied: Diabetic kidney disease (DKD) is one of the most common complications of diabetes mellitus. Jingui Shenqi pills (JSP) is a Chinese patent medicine, It is widely used in China to treat DKD. At present, there are some reports about the treatment of DKD with 
JSP, but the conclusions are not consistent, However, there is no evidence-based medical evidence to prove its safety and effectiveness, and most of the research samples are small, which can not provide enough test efficiency. The purpose of this systematic review is to evaluate the efficiency and safety of JSP in the treatment of DKD.

\section{METHODS}

Participant or population: Participants were those who had been diagnosed as diabetic kidney disease regardless of age, gender, nationality and race.

Intervention: The experimental group used astragalus injection combined with Jingui Shenqi pillsThe experimental group used astragalus injection combined with Jingui Shenqi pillsThe experimental group used astragalus injection combined with ACEI/ ARB treatment.

Comparator: The control group used another active treatment or placebo or no treatment. The dosage and frequency will not be restricted.

Study designs to be included: All RCTs about JSP for DKD will be included regardless of language. The following studies will be excluded:animal experiments, case series, quasi-RCTs, cell experiments, Case reports, non-RCTs.

Eligibility criteria: Studies recruiting patients with DKD, as diagnosed with any recognized diagnostic criteria, will be included in our study, regardless of gender, ethnic background or nationality. However, subjects with infection, fever, cancer, kidney transplantation, liver disease, and severe cardiopulmonary disease will be excluded.

Information sources: We will search the following databases from their inception to October 30, 2021: EMBASE Database, Web of Science, PubMed, the Cochrane Library, China National Knowledge Internet, Wanfang Database, China Biological
Medicine Database, VIP database, and Ongoing Clinical Trials Database.

Main outcome(s): The primary outcome measures set in our study included urinary albumin excretion rates, 24 hours urinary protein quantification, and renal function(blood urea nitrogen, serum creatinine concentration).

Data management: Each of the included RCTs needs to be assessed for risk of bias, which was done independently by the two authors using the Cochrane Risk of bias tool, and the disputed part resolves the disagreement through negotiation or a third author.

Strategy of data synthesis: We performed this meta-analysis using Revman 5.4 software (Cochrane Collaboration) for all statistical data analyses, using 95\% confidence interval( $\mathrm{Cl}$ ) and risk ratio (RR) to calculate categorical variables, and using $95 \% \mathrm{Cl}$ and mean differences (MDs) to calculate continuous variables. We used the $X^{2}$ value test and inconsistency index $\left.{ }^{(}{ }^{2}\right)$ to assess the heterogeneity across each study. A value of $P<0.1$ or $I^{2}>50 \%$ was deemed to have significant heterogeneity, a random-effect model was then used to analyze the data. Otherwise, the fixedeffect model was used. We adopted the Egger funnel plot and Egger's test to test publication bias with Stata15.0 (StataCorp LP, College Station, TX, US), with $P<0.05$ indicating significant asymmetry.

Subgroup analysis: We will investigate the source of heterogeneity using subgroup analysis based on different interventions, controls, and outcomes. Subgroup analysis was used to find the potential sources of heterogeneity.

Sensitivity analysis: We will carry out a sensitivity analysis to investigate the robustness and stability of outcome results by removing low methodological quality studies. The main analysis points included the impact of method quality, sample size, and missing data on the study.

Country(ies) involved: China. 
Keywords: Jingui Shenqi Pills; diabetic kidney disease; meta-analysis.

Contributions of each author:

Author 1 - Pingjie Xiong.

Author 2 - Xinyu Liu.

Author 3 - Xiaoqiang Huang.

Author 4 - Weifen Fang.

Author 5 - Shenghua Piao.

Author 6 - Jiao Guo. 